\title{
Automated behavioral experiments with individually marked bees
}

\author{
R. MENZEL and U. GREGGERS \\ Freie Universität Berlin, Berlin, Federal Republic of Germany
}

\begin{abstract}
Two automated techniques for investigating appetitive conditioning in honeybees are described. In the first method, free-flying bees conditioned to color marks are detected automatically at their approach to a color target. The second method allows the detection and separation of individually marked bees. At least 10 different bees can be processed and differentially detected at the same time. Each bee may control its own reward and test procedure. Computeraided data analysis and experimental procedures are described. Illustrative experiments on conditioning and choice behavior are reported.
\end{abstract}

A central problem in studying the behavior of honeybees is response detection. Bees are fast flyers and approach a target in quick turns and advances. When they have landed on a target, their dipping into a hole can be detected by a photocell (Grossmann, 1973; Sigurdson, 1981 ), or their contact with the substrate can be detected by a capacitance device (Sigurdson, 1981). But these automated procedures have limitations in that (1) bees cannot be detected during flight, and (2) the identification of individual subjects is made by the experimenter. One of the purposes of this paper is to describe a method for measuring the approach to a target by free-flying bees, a method that previous observations have shown to be a sensitive indicator in training experiments (Menzel, 1967). A second purpose is to describe an identification method for individually marked bees that permits the entire experiment to be controlled by a computer.

\section{AUTOMATED DETECTION OF THE APPROACH OF FREE-FLYING BEES TOWARD A VERTICALLY ORIENTED TARGET}

A visual target (in this case, a round disk of diameter $7 \mathrm{~cm}$, with half-sectors of different brightness or hue) commonly used in training is depicted schematically in Figure 1. Bees learn to associate this target with a sucrose solution. Three of these targets are positioned at equal distances from each other on a board. The target in the center is for training, and the two others are the test targets. During training, only the center target is displayed. During testing, the training target is covered with a white plate, and the two other targets are shown. Figure 1 shows

Information on the software and computer technology is available from the senior author, whose mailing address is: Freie Universitat Berlin, Institut für Tierphysiologie, Grunewaldstrasse 34, 1000 Berlin 41, Federal Republic of Germany. a test target. In the training target, the centrally located tube (T) gives free access to a container with sucrose solution; in the test targets, red filters prevent the bees' entering the tube. Red is invisible to the bee, so it mimics a dark hole. Figure 1 shows three photodiodes (PD) arranged in the tube behind the red filter (RF). The photodiodes are pointed towards a crosspoint about $1 \mathrm{~cm}$ in front of the target. When a bee approaches the target and reaches the crosspoint, it screens off the background light. This causes an abrupt onset of the photodiode signal. The signals from the photodiodes at the two alternative targets during a test are shown in Figure 2a. These voltage fluctuations are converted into a frequency modulation and are stored on tape. A third trace of the tape carries information on the test situation and informs the computer which target was positioned on which side and, hence, which data of successive tests should be assigned to the right (training) or wrong (alternative) target. A fourth trace stores the experimenter's verbal protocol.

The computer (Nicolet Med 80) is programmed to count all impulses above a certain threshold (called "counts"), and stores all counts separately for each of the two alternatives. Statistical tests are run by the computer consecutively, according to the specific test situation. A useful way of displaying the data is to have the computer calculate the temporal sequence of the counts of a single experiment or of all identical experiments. Such a histogram is shown in Figure 2b. About two-thirds of all counts are separated by less than 2 sec. Comparison with experimenter judgment has shown that separate approaches are counted if the animal leaves the target for at least $2 \mathrm{sec}$. This means that, on the average, each approach of a bee to the target is coded by two or three counts because the animals hover in front of the target.

A comparison of three kinds of data evaluation is shown in Figure 3 for a specific experiment on discrimination between color patterns. The three kinds of 


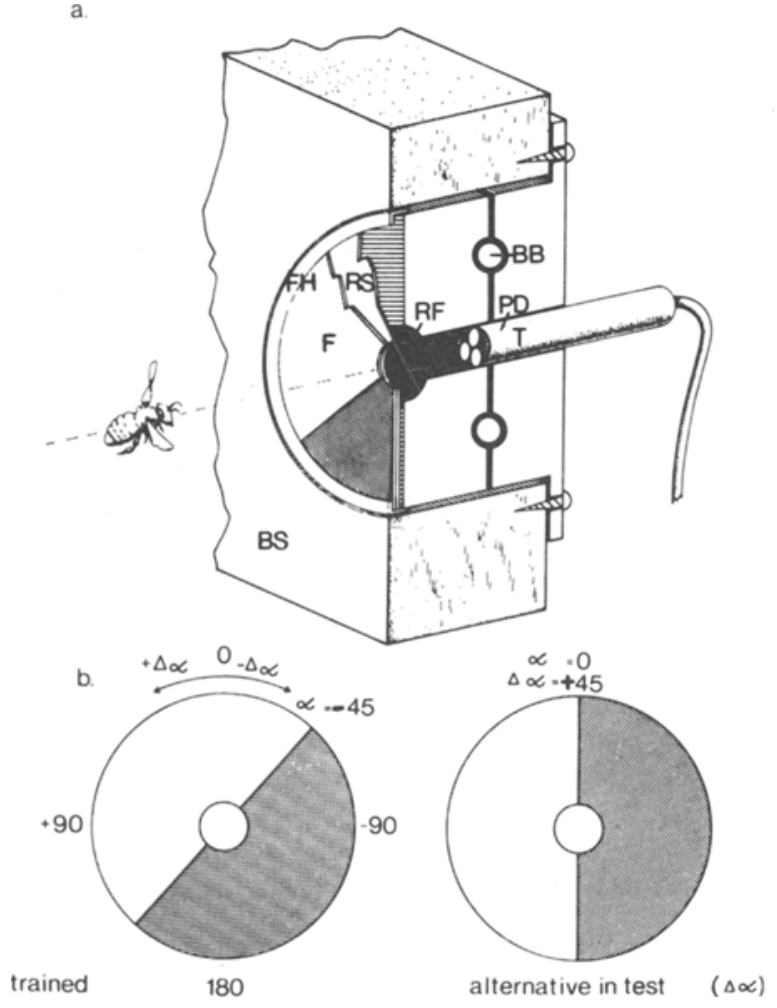

Figure 1. (a) Arrangement of a test target for freely flying bees. Three of these devices are mounted on a vertical board (BS; $2 \times 2 \mathrm{~m}$ ). The device used during training is positioned in the middle of the board and differs from the one depicted here by the lack of the three photodiodes (PD). In this case, bees reach a sucrose solution by entering the tube $(T)$ in the middle of the color pattern [filter $F$ on a reflecting screen (RS)]. During the test situation, the training device is covered with a white plate, and two devices of the kind shown here appear right and left at equal distances from it. These devices contain a red filter (RF), which covers the entrance to the tube, and the three photodiodes (PD). Any directed approach of the test bee is monitored by the photodiodes. (BB: ball bearings, which allow the testing of any rotational position of the color pattern; FH: filter holder.) (b) Example of training and test targets, which are composed of two half sectors differing in hue. In this case (see also Figure 3), the bees are trained to an ultraviolet half-sector (white) and a blue-green half-sector (stippled); the contrast line between the sectors is tilted 45 deg to the right ( $\alpha=-45 \mathrm{deg}$ ). In the test situation, the trained pattern and the alternative pattern are shown. The difference between the trained pattern and the alternative pattern is expressed in the deviation of inclination of the contrast line $(\Delta \alpha)$.

data evaluation are: choices counted by the experimenter, all counts by the photodiode device, and counts by the photodiode device that are separated by at least 2 sec. In the experiment shown in Figure 3, the training color pattern is composed of an ultraviolet-reflecting half-sector and a blue-green-reflecting half-sector, with the contrast line between the two colors inclined $40 \mathrm{deg}$ to the right. The alternative patterns in the consecutive tests are of the same color pair, but with different orientations of the contrast line (compare Figure 1 and the abscissa of Figure 3). The highest response values are reached by the choice judgment of the experimenter. The number of counts, however, is much lower than that of the two automated counts. The Kolmogoroff-Smirnow test shows that percentage differences of $\geqslant 9 \%$ are significant $(p \leqslant .01$ level) for all automated counts. For the experimenter's judgment, the number is $\geqslant 15 \%$; with automatic counting eliminating all counts closer together than $2 \mathrm{sec}$, the number is $\geqslant 17 \%$. Thus, it appears that the totally automatic counting is the most sensitive measure and offers the best compromise.
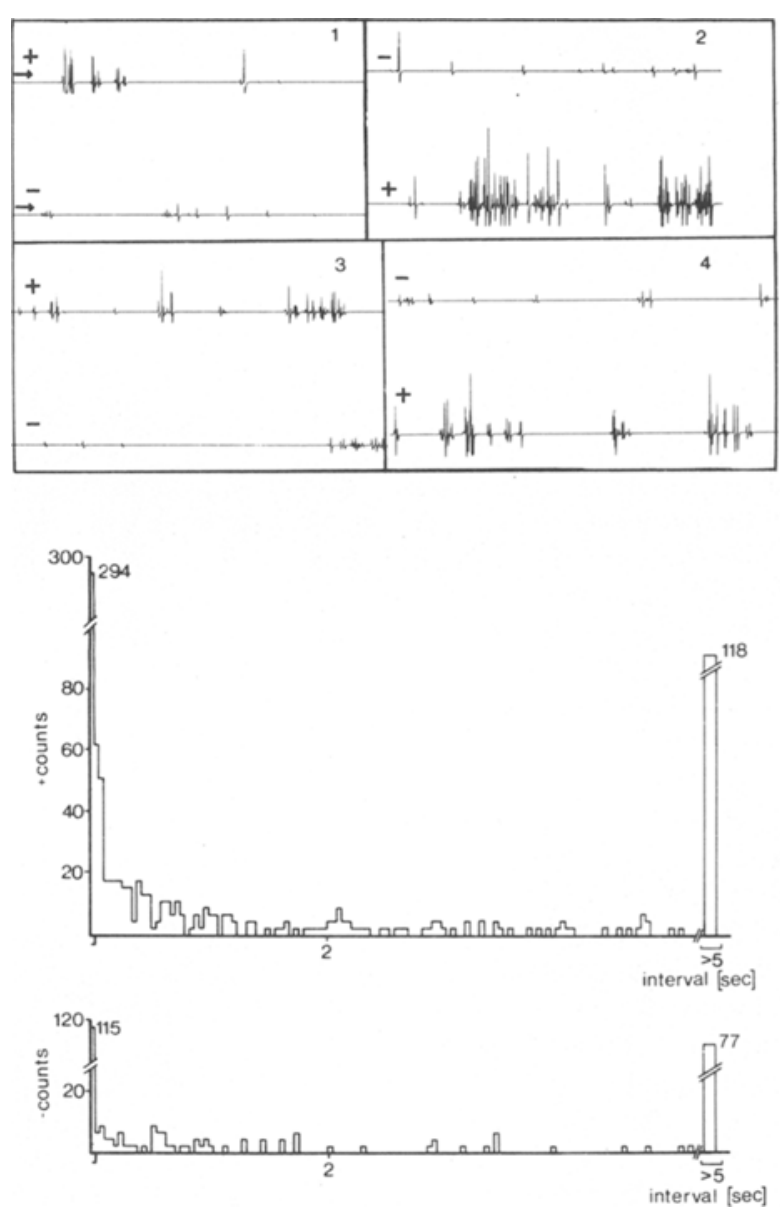

Figure 2. (a) Signals from the photodiodes of the two test devices (Figure 1) during a 4-min test period. + and - indicate the trained and alternative color pattern, respectively. The position of the two color patterns is changed every minute $(1,2$, $3,4)$. The training pattern in this case is composed of half a circle of uitraviolet and half a circle of blue-green (see Figure 1b); inclination of the contrast line is $45 \mathrm{deg}$ to the vertical $(\alpha=-45 \mathrm{deg}$ ). The alternative pattern during the test is composed of the same colors, but at a different inclination of the contrast line $(+45 \mathrm{deg})$. Two bees are flying throughout the test. The arrows on the left upper side mark the threshold for the counting by the computer. (b) Example of an interval histogram for the training pattern (upper histogram, see Figure $2 b$ ) and the al ternative pattern (lower histogram). The histograms are calculated from nine different 4-min tests. All intervals shorter than $40 \mathrm{msec}$ are sampled in the first bin (bin width $=40 \mathrm{msec}$ ); all intervals longer than $5.08 \mathrm{sec}$ are sampled in the last bin (see text). 


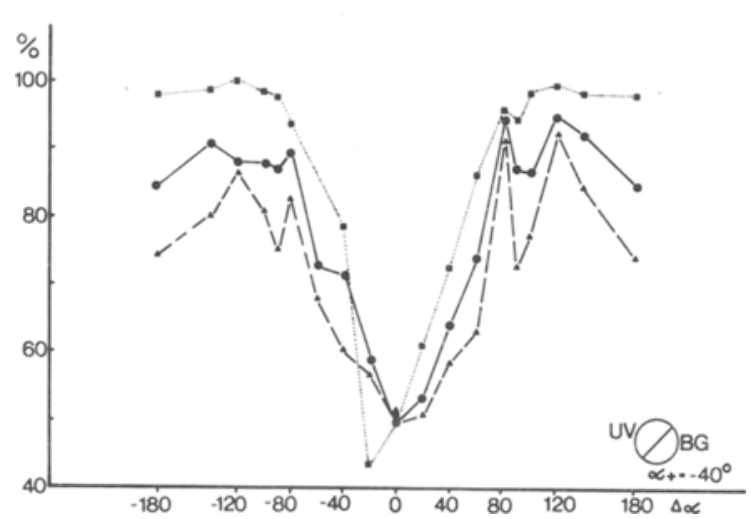

Figure 3. Comparison of three different kinds of choice measurement. The ordinate gives the choice reaction in percentage of correct choices. In this experiment, bees are trained to an ultraviolet/blue-green pattern, with an inclination of the contrast line of $\alpha=45 \mathrm{deg}$ (see Figures 1b, 2a, and 2b). The alternative pattern in the test is composed of the same colors, but inclination $(\Delta \alpha)$ is varied in successive tests (abscissa), $(-\Delta \alpha$ : rotation clockwise; $+\Delta \alpha$ : rotation anticlockwise, Figure 1b). At $\Delta \alpha=$ 0 , the alternative pattern is the same as the training pattern; choice reaction (ordinate) is therefore $50 \%$ (equal choices to both alternative patterns). In the curve $\bullet-\bullet$, all detections by the detection device are used (see Figure 2b); total number = 16,952 ; in curve $\wedge--\backsim$, only those detections from the detection device that are separated by more than $2 \mathrm{sec}$ are used; total number $=3,528$; in the curve $\bullet . . .$. , choices that were counted by the experimenter are used; total number $=1,717$ (see text).

\section{AUTOMATIC DETECTION OF INDIVIDUALLY MARKED BEES}

The automatic recognition of an individual bee that is flying freely or is running in a tube is a difficult technical problem, because the position of the body in space changes rapidly and markedly. This makes it virtually impossible to use optical methods. We have developed a technique that allows an investigator to recognize at least 10 differently marked bees. For marking bees, a stainless steel ball (produced for ball bearings) of diameter $0.7-1.7 \mathrm{~mm}$ (weight $=1.3-18 \mathrm{mg}$ ) is glued to the thorax. The gluing method is critical. We use a dentist's stone polisher to gently grind off the wax layer, and we immediately fix the ball with an acrylic glue under a stereomicroscope. This procedure marks the bee reliably for weeks. Bees can be loaded with excess weight of up to $55 \mathrm{mg}$ without their behavior being changed (Gontarski, 1935; Heran, 1963). The frequency of the foraging flights is even increased by the additional load.

The different sizes of the stainless steel balls are detected by the following physical principle: Ferromagnetic material brought into an electromagnetic field reduces the frequency of that field, because the inductivity of the oscillating circuit increases. The amount of the frequency change is a function of the volume and magnetic permeability of the ferromagnetic material brought into the field.
To be detected, bees have to be made to run through a tube (Figures 4 and 5). This is achieved easily by first training the bees to approach the entrance to the tube and then to go through the tube by slowly moving the feeder with the sucrose solution stepwise through the tube. The diameter of the tube is $15 \mathrm{~mm}$, which is twice the height of a running bee. The stainless steel ball is, therefore, always in the center of the tube, independently of the direction and orientation in which the bee chooses to walk. The detector coil is wound around a 90-deg sector of a sphere in a crossed fashion and surrounds a segment of the tube at positions at which the bees are to be detected $\left(D_{E}, D_{L}\right.$, and $D_{R}$ in Figure 5). The frequency change induced in the coil by a bee running through the tube depends on the accuracy of the central alignment of the stainless steel ball. A deviation of $\pm 4 \mathrm{~mm}$ from the center of the 15 -mm-diam tube causes a $2 \%$ deviation. More than $95 \%$ of all runs aligned better than $\pm 4 \mathrm{~mm}$ to the central axis of the tube. The frequency change is measured by a microprocessor counting several periods of the oscillations. Changes below a threshold are interpreted as

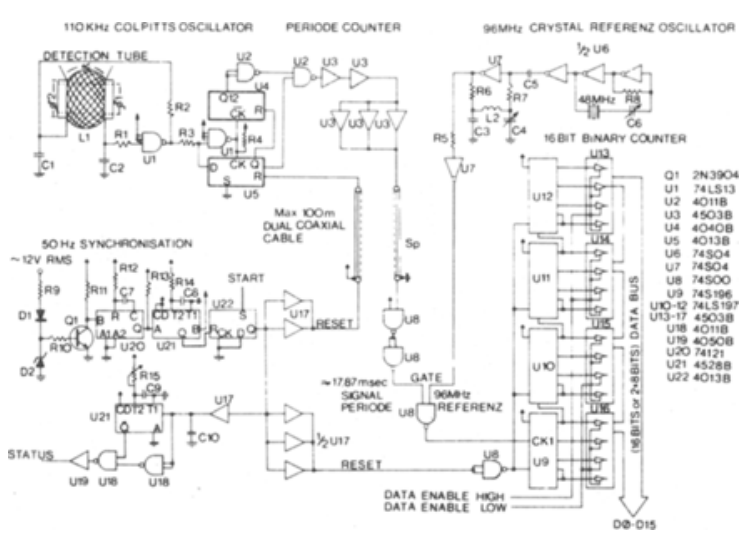

Figure 4. Circuit diagram of the device for detection of individually marked bees. The wiring diagram includes all information necessary to build one's own device. The marked bees run through a tube with the inner diameter 1 and outer diameter 2. The symbol $\alpha$ defines the sector of a sphere (in this case $90 \mathrm{deg}$ ), on top of which a coil $(\llcorner 1)$ with 100 turns of No. 38 AWG is placed. The detection tube, the 110-kHz Colpitts oscillator, and the period counter are mounted in a metal box and connected by a dual coaxial cable (up to $100 \mathrm{~m}$ ) with the interface. The interface receives the integrated signal through the SP line. The 96-MHz reference oscillator is used for the measurement of the duration of the signal period. The result of the 16-bit binary counter is shifted to a microprocessor (not shown here) through the data bus. The whole measuring procedure is synchronized by the line frequency $(50-\mathrm{Hz}$ synchronization, left lower side) to reduce any line-noise interference. The connections status, data enable high, data enable low, and start are under the control of the microprocessor. The job of the microprocessor is to read the instantaneous frequency of the detector through the 16-bit data bus, reject artifacts, separate baseline fluctuations from signals, and detect the maximal frequency change during a signal. The result corresponds to the volume of the ferromagnetic markers and identifies a given individual bee. This number is transferred to a table computer, together with the accurate time and a code for the detection tube. 


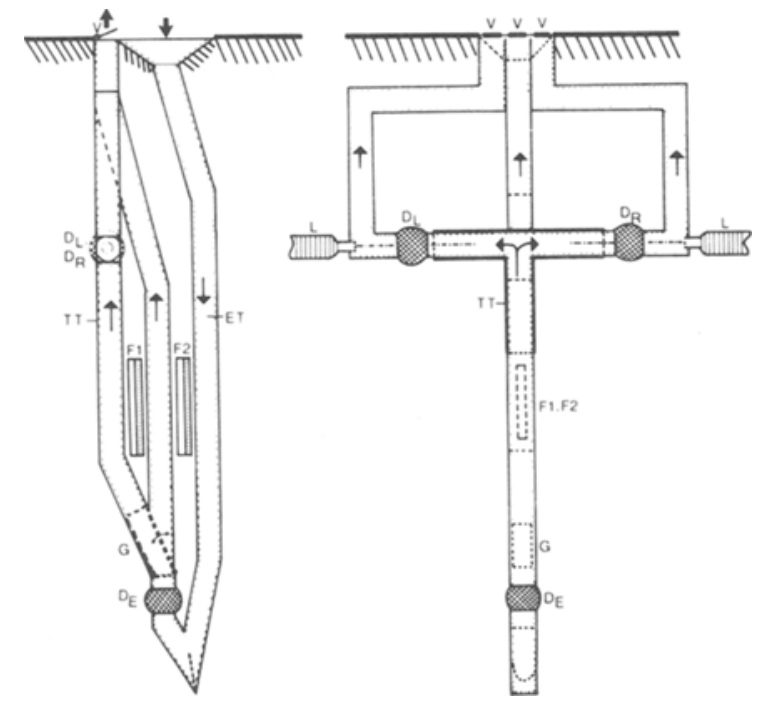

Figure 5. Experimental arrangements of the phototaxis experiment. The tubes are arranged horizontally. The diagram to the left is a side view; to the right is a top view. Bees enter the tube system at the arrow pointing inward. After running through the entrance tube (ET), they reach a detection coil $\left(D_{E}\right)$ where marked bees are separated from unmarked bees by a movable sector of the whole tube (G). Marked bees are directed into the upper tube (TT); unmarked bees reach the middle tube. Marked bees feed on sucrose solution at F1; unmarked bees feed at F2. The exit for both the marked and the unmarked bees is indicated by the outward-pointing arrow. The marked bees run into a T-maze (test tube, TT) on their way outwards and make a choice according to their positive phototactic tendency. $D_{L}$ and $D_{R}$ are detecting tubes at the end of the $T$-maze; $L$ indicates flexible light guides through which the spectral light stimuli or mixtures of spectral light are presented.

random deviations and are used to set a new baseline. Above the threshold, the microprocessor stops the baseline adjustment and looks for the maximum of the frequency change. This value is proportional to the size of the stainless steel ball. Figure 4 gives the technical details of the microprocessor-controlled system.

If animals other than bees are to be detected, the diameters of the coil and the tube have to be altered. The tube diameter should be twice the height of the animal. The running speed of the animal determines the optimal sample frequency, because at least three sample points are needed to detect the peak of the frequency change.

Integration time (IT) should be: IT $=\mathrm{S} / \mathrm{V}_{\max }$, where $S$ is defined as the running height of the animal and $\mathrm{V}_{\max }$ is defined as its maximal running speed. Besides $\mathrm{V}_{\text {max }}$, the critical parameter is the length of tube within the detector coil. Since the detector coil is spheri$\mathrm{cal}$, the center length equals the radius of the tube, which is also the height of the animal.

The sensitivity of the volume detection of the stainless steel ball is defined by: $\Delta V_{\text {ferro }}=(6 / \mu)(\Delta t / I T)$ ( $\mathrm{V}_{\text {coil }}$ ), where $\Delta \mathrm{V}_{\text {ferro }}$ is the smallest volume of the ferromagnetic material to be discriminated, $\mu$ is the effective permeability of the ferromagnetic material (1.8-1.9 for stainless steel balls), $\Delta t$ is the shortest detectable time period (10 nsec for a $100-\mathrm{MHz}$ frequency and a $100-\mathrm{MHz}$ counter), and IT is the integration time. The factor of 6 results from the proportionality between change in inductivity and frequency (factor of 2) and inductivity and volume change of the ferromagnetic material (factor of 3). We find for the bee a total range

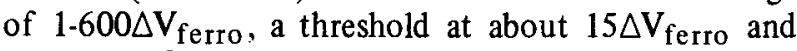
a scatter of $\pm 10 \%$, which means that at least 10 differently marked bees can be reliably detected.

\section{EXAMPLE OF AN EXPERIMENT: PHOTOTAXIS OF DARK-ADAPTED BEES}

In an attempt to study the question of whether bees use colors in their spontaneous phototactic behavior, dark-adapted bees have to be tested in the dark with respect to their phototactic choice of spectral lights and mixtures of spectral lights. In such an experiment, it is important to test individual bees because of their individual differences and the necessity of determining exactly the status of dark adaptation by measuring the light intensity outside and the time the bee has been in the dark before having to make the choice reaction.

Bees are trained to enter a tube that guides them to a feeding place (ET in Figure 5). Halfway in, the bees run through a detecting tube $\left(D_{E}\right)$ that separates marked bees from unmarked bees by means of a movable section of the tube (G). When a specifically selected marked bee is detected, the computer gives access to the upper part of the tube system (TT). Which of the marked bees-all, several, or only one-is selected is controlled by software. The unmarked bees feed on sugar water at F2 and leave the tube system through the middle exit (V). The selected marked bee(s) is fed to satiation at F1, after which it is positively phototactic and chooses the illuminated arm of the T-maze (TT). The choice is detected by one of the detecting tubes $\left(D_{L}\right)$, and the bee leaves the tube system through the right or left exit (V). Whenever the computer registers a detection in the $D_{E}$ or $D_{L}$ tubes, it also reads off the date, time of day, temperature, and light intensity outside. Before the data are stored on tape, the computer assigns a number to the bee and looks at the recent history of the bee. In this particular experiment, we programmed the computer to reconstruct the whole circle of the last foraging flight. Redundant data and choices after a too-short dark-adaptation time are specially assigned. Computeraided data evaluation from the tape-stored data allow various kinds of calculations, such as histograms of the detection values, or dependence of choice reaction on light intensities, on wavelength of light, on ambient light intensity outside, on dark adaptation time, on individual differences, and so forth. This device also has been used in conditioning experiments, in which the whole experiment was controlled by the computer. Following a program, the computer selects visual stimuli, 
performs a training or a test trial, excludes or includes particular bees, and controls the feeding of the unmarked bees.

\section{DISCUSSION}

The two devices reported here have been compared very accurately with the observations of an experienced but uninformed observer. In addition, comparison was also made on the basis of a statistical evaluation of the number of data collected and the scatter of the data. The results indicate that both automatic methods are not inferior in reliability, objectiveness, and efficiency when compared with an observer, and that they offer obvious additional advantages.

In the case of the approach detector for free-flying bees, the duration of a choice can be detected at high accuracy by measuring the time between the first and last count of a continuous sequence of counts. In experiments with only one bee flying in a choice experiment, the temporal sequence between choices of the right and wrong target can be analyzed.

The automatic detection of individually marked bees allows the researcher to collect information from many detecting tubes at the same time. We are measuring at four places, but the number can be increased easily. We have measured flight duration between hive and feeding place to millisecond accuracy. Flight activity during the day has been monitored for individual bees over a period of weeks. In an experiment with young bees, their first flight activities were monitored.

Another possible use of the method is to control the experimental procedure by the computer according to a computer program, which sets different experimental conditions for different marked bees. For example, several different bees are run on different training and test procedures at the same time. The computer controls access to the tube system, decides whether a bee performs a training or test trial, and adjusts the quantity of food reward according to the speed of running of the test bee. There is another advantage, which should not be underestimated. In summer, bees may start flying as early in the morning as 4:30 a.m. and may continue to fly until 10 p.m., weekends included. The apparatus allows the user to extend considerably the observation time, and to record the behavior of the test animals without interruption.

If smaller animals (ants, flies, beetles) have to be detected, the detector tube has to be adjusted according to the formula given in the text. Smaller tubes have a higher sensitivity, and ferromagnetic material of less than $1 \mathrm{mg}$ is detectable.

\section{REFERENCES}

Gontarsk I, H. Leistungsphysiologische Untersuchungen an Sammelbienen. Archiv fur Bienenkunde, 1935, 16, 107-126.

Grossmann, K. E. Continuous, fixed-ratio, and fixed-interval reinforcement in honey bees. Journal of Experimental Animal Behavior, 1973, 20, 105-109.

Heran, H. Wie beeinflußt eine zusätzliche Last die Fluggeschwindigkeit der Bienen. Zoologischer Anzeiger, 1963, 26, 346-354.

Menzel, R. Das Erlernen von Spektralfarben durch die Honigbiene. Zeitschrift für vergleichende Physiologie, 1967, 56, 22-62.

Sigurdson, J. E. Measurement of consummatory behavior in honey bees. Behavioral Research Methods \& Instrumentation, $1981,13,308-310$.

(Manuscript received October 7, 1982; revision accepted for publication February 22, 1983.) 